\title{
Chronic pelvic pain in urogynecological practice
}

\author{
Peter L. Dwyer
}

Published online: 24 February 2011

(C) The International Urogynecological Association 2011

In this issue of the International Urogynecology Journal there is a focus on chronic pain in urogynecological practice. Kavvadias et al. performed a literature review of definitions and assessment of chronic pelvic pain in the urogynecological patient, and there are a number of original manuscripts relating to chronic pelvic pain conditions including interstitial cystitis and pain after sling surgery. We believe that this is timely as referral of women with chronic pelvic pain is a common occurrence for all of us. Some of these women will have pain which is related to the lower urinary tract with urinary frequency, urgency, and difficult or painful micturiction and possibly have a provisional diagnosis of interstitial cystitis or urethral syndrome. Others may have predominantly genital or colorectal pain which may be constant or intermittent, or related to coitus or defecation. The pain may have developed spontaneously or following your or somebody else's surgery. Many of these women may have been diagnosed as having pudendal neuropathy or entrapment, pelvic congestion syndrome, fibromyalgia, vulva vestibulitis, and irritable bowel syndrome. Women with chronic pelvic pain frequently have dysfunctional sexual symptoms as well as emotional and psychosocial problems, and sometimes childhood sexual or physical abuse.

In women with chronic pelvic pain where there is no clearly defined pathology, accurate diagnosis is difficult as the underlying aetiology is unclear. There is a lack of diagnostic investigations for many pelvic conditions and treatment strategies are often unsuccessful. These patients have frequently seen multiple doctors and finally in desperation referred to you, a urogynecologist or urologist because of associated urinary symptoms. Not surprising, medical ligation is common in this group of patients especially if considered to be surgically related. This tends to perpetuate the pain and complicate management.

Therefore, considering the importance of chronic pain in urogynecology, we would encourage good innovative research and prospective trials, and the submission of original articles and reviews in this area to the International Urogynecology Journal.

P. L. Dwyer $(\bowtie)$

Department of Urogynecology, Mercy Hospital for Women,

University of Melbourne,

Melbourne, Australia

e-mail: pdwyer@connexus.net.au 